\title{
INVESTIGAÇÕES GPR NOS DISTRITOS MINEIROS DE SANTA BÁRBARA E BOM FUTURO: PROVÍNCIA ESTANÍFERA DE RONDÔNIA
}

\author{
Jorge Luís Porsani ${ }^{1}$, Carlos Alberto Mendonça ${ }^{2}$, Jorge Silva Bettencourtt ${ }^{3}$, Francisco Yukio Hiodo ${ }^{4}$, \\ José Antonio Jerônymo Vian ${ }^{5}$ e Jorge Eduardo da Silva ${ }^{6}$ \\ Recebido em 19 janeiro, 2004 / Aceito em 21 julho, 2004 \\ Received January 19, 2004 / Accepted July 21, 2004
}

\begin{abstract}
GPR - Ground Penetrating Radar investigations were carried out on Santa Bárbara and Bom Futuro Mining Districts (The Rondônia Tin Province). The main purpose of this study was to check a new application of this method directed towards the detection of primary and secondary tin deposits, and to optimize detailed exploration costs. The GPR profiles were acquired with antennas of 25,50 and $100 \mathrm{MHz}$, that allowed to investigate the subsurface down to 30 meters depth. Greisen bodies at the Santa Bárbara and Bom Futuro primary tin deposits were easily detected through the GPR survey. In Bom Futuro mining district, a paleochannel structure at the bottom of the alluvial sedimentary sequence was also identified. The geophysical results show an excellent agreement with borehole log geological informations, which prove the relevance of this new application of GPR method for tin exploration. It is suggested that this method might be successfully applicable to similar geological environments within the Amazon Cráton. Moreover, the exploration expenditures might be optimized if the GPR investigations were conducted before drilling campaign.
\end{abstract}

Keywords: GPR - Ground Penetrating Radar - Georadar Geophysical Prospecting, Primary and Secondary Cassiterite Deposits, Santa Bárbara and Bom Futuro Mining Districts, Rondônia Tin Province.

RESUMO. Investigações GPR - Ground Penetrating Radarforam realizadas nos distritos mineiros de Santa Bárbara e Bom Futuro (Província Estanífera de Rondônia), visando averiguar a aplicabilidade do método para localização de depósitos estaníferos primários e secundários, bem como otimizar os custos envolvidos na etapa de prospecção. Os perfis de reflexão GPR foram adquiridos com antenas de 25,50 e $100 \mathrm{MHz}, 0$ que permitiu investigar a subsuperfície até 30 metros de profundidade. Em relação aos depósitos primários das minas de Santa Bárbara e de Bom Futuro, o método GPR permitiu identificar zonas com predominância de corpos de greisens. No depósito secundário aluvionar da mina de Bom Futuro, o método GPR permitiu localizar uma estrutura de paleocanal na base do sistema deposicional sedimentar. Os resultados obtidos apresentaram uma excelente concordância com as informações geológicas provenientes das sondagens, demonstrando essa nova aplicabilidade do método GPR para a exploração mineral, na qual poderá ser extendida para ambientes similares do Cráton Amazônico. Além disso, os custos envolvidos no processo exploratório poderão ser otimizados, caso as investigações GPR sejam realizadas antes da execução de sondagens.

Palavras-chave: Prospecção Geofísica GPR - Ground Penetrating Radar - Georadar, Depósitos Primários e Secundários de Cassiterita, Distritos Mineiros de Santa Bárbara e Bom Futuro, Província Estanífera de Rondônia.

${ }^{1}$ IAG-USP, Departamento de Geofísica, Rua do Matão 1226, Cidade Universitária, 05508-090 São Paulo, SP - Tel: 0xx11 30914734 - Fax: 0xx11 30915034 E-mail: porsani@iag.usp.br

${ }^{2}$ IAG-USP, Departamento de Geofísica, Rua do Matão 1226, Cidade Universitária, 05508-090 São Paulo, SP - Tel: 0xx11 3091-4751 - Fax: 0xx11 30915034 E-mail: mendonca@iag.usp.br

${ }^{3}$ IGc/USP, Instituto de Geociências, Rua do Lago, 562, Cidade Universitária, CEP 05508-080 São Paulo, SP - Tel: 0xx11 30914205 - Fax: 0xx11 30914295 E-mail: jsbetten@usp.br

${ }^{4}$ IAG-USP, Departamento de Geofísica, Rua do Matão 1226, Cidade Universitária, 05508-090 São Paulo, SP - Tel: 0xx11 30914777 - Fax: Oxx11 30915034 E-mail: francisc@iag.usp.br

${ }^{5}$ CESBRA S.A. BR-364, Km 601,5 (zona rural), CEP 78937-000, Município de Itapuã do Oeste, Rondônia - Tel: 0xx69 2231069 - Fax: 0xx69 2244371 E-mail: jaj@@cesbra.com.br

${ }^{6}$ IAG/USP, Graduação em Geofísica, Rua do Matão, 1226, Cidade Universitária, 05508-090 São Paulo, SP - Tel: 0xx11 30914775 - Fax: $0 x x 1130915034$ E-mail: quartafeira@hotmail.com 


\section{INTRODUÇÃO E OBJETIVOS}

A cassiterita foi descoberta no Estado de Rondônia em 1952 e, durante a década de 60 , sua extração foi realizada por garimpeiros (Bettencourt et al., 1997). Em 1971 o governo federal lançou um programa de exploração mineral visando avaliar o potencial de estanho de Rondônia. Como resultado, foi criada a Província Estanífera de Rondônia a qual compreende uma área de aproximadamente $87.000 \mathrm{~km}^{2}$ dentro do Estado de Rondônia e parte dos Estados do Amazonas, Acre e Mato Grosso do Sul.

De 1959 a 1984, a Província Estanífera de Rondônia contribuiu com $78,5 \%$ do total da produção brasileira de estanho. Entretanto, a partir de 1985, com a queda do preço desse metal no mercado internacional, a maioria das minas foi fechada, embora a produção, nessa província, tenha aumentado em 1987 devido à descoberta do distrito mineiro de Bom Futuro.

Um excelente histórico sobre a mineração e 0 garimpo de Cassiterita em Rondônia, até 1996, pode ser encontrado em Dall'Igna (1996). De 1997 até o presente momento, dois distritos mineiros encontram-se em operação, as minas de Santa Bárbara e de Bom Futuro, cuja produção anual de 7.500 ton em 1997, colocaram 0 Estado de Rondônia como 0 segundo produtor brasileiro desse metal. Os distritos mineiros de Santa Bárbara e de Bom Futuro estão situados nos municípios de Itapuã do Oeste e de Ariquemes, Estado de Rondônia, região noroeste do Brasil (Figura 1).

As duas principais feições geológicas importantes à prospecção de Cassiterita na Província Estanífera de Rondônia são as fácies greisenizadas, estruturas tipicamente encontradas nos depósitos primários, encravadas em cúpulas graníticas, e os paleocanais internos às seqüências deposicionais que, via de regra, são mais ricos em cassiterita, e que em parte constituem os depósitos secundários (Leite Jr., 2002). A atividade exploratória é mais dirigida à procura de estruturas do tipo greisens, que podem apresentar teores de Sn economicamente atraentes, além dos paleocanais que podem exibir, altas concentrações estaníferas. Normalmente, as estruturas do tipo greisennão chegam até a superfície, e por essa razão, a sua busca tem sido realizada pelo uso intensivo de sondagens, elevando bastante os custos da exploração e 0 impacto ambiental.

As Investigações GPR-Ground Penetrating Radar, ora realizadas nos distritos mineiros de Santa Bárbara e de Bom Futuro (Província Estanífera de Rondônia), tiveram como objetivos averiguar, não só a aplicabilidade do método para a localização de depósitos estaníferos primários e secundários, como também, a otimização dos custos envolvidos nas eta- pas de prospecção e avaliação de depósitos. Concomitantemente, outras investigações geofísicas também foram realizadas, utilizando-se os métodos da eletrorresistividade, caminhamento eletromagnético, gravimetria, magnetometria e espectrometria gama (Mendonça, 2000). As investigações geofísicas foram realizadas em locais previamente investigados por sondagens ao longo de perfis, permitindo, assim, comparar os resultados geofísicos e geológicos.

Os perfis GPR foram realizados nos depósitos primários das minas de Santa Bárbara (Cortez I) e de Bom Futuro e nos depósitos secundários aluvionares de Santa Bárbara (Taboquinha). Embora outras investigações geofísicas fossem realizadas sobre os mesmos perfis, os resultados das investigações GPR, de um modo geral, foram conclusivos e compatíveis com os dados de sondagens, justificando assim, a sua apresentação de forma isolada neste artigo.

\section{DEPÓSITOS PRIMÁRIOS E SECUNDÁRIOS DE ESTANHO}

Os depósitos primários são caracterizados pela presença de greisen (in-sitt) no maciço ou quartzo greisens relacionados com diques de rochas ácidas como riolito/quartzo pórfiro (tipo stockwork ou enxame de veios). Essa rocha resulta da alteração hidrotermal do granito in-situ, após ou concomitante a eventos tectônicos como fraturas e falhas. No geral, a relação de contato com a rocha encaixante é brusca e bem definida, ocorrendo de vertical a subvertical. Como exemplo deste tipo de depósito tem-se a mina de Santa Bárbara. A Figura 2 mostra um perfil geológico obtido a partir da correlação litológica entre sondagens no Depósito Primário de Cortez, Distrito de Santa Bárbara. Os depósitos primários constituem as áreas fonte da cassiterita que se acumula nos depósitos secundários.

Os depósitos secundários são originados da intensa decomposição química e desagregação mecânica da rocha granítica original ou da rocha greisenizada. A cassiterita é um mineral resistente à intemperização e pode ser encontrada na forma residual (in-sitt) nos elúvios, na forma semitransportada nos colúvios e na forma transportada nos alúvios. No depósito do tipo coluvionar, as rochas depositam-se nas encostas e planícies próximas ao afloramento do depósito primário. Como exemplo deste depósito podemos citar os depósitos Taboquinha e Cortez, ambos detríticos. A Figura 3 mostra um perfil geológico dos depósitos primário e aluvionar, na mina de Bom Futuro e, a Figura 4 mostra um perfil geológico do depósito aluvionar de Taboquinha, na mina de Santa Bárbara. Estes perfis geológicos foram obtidos a partir da correlação litológica entre as sondagens. 


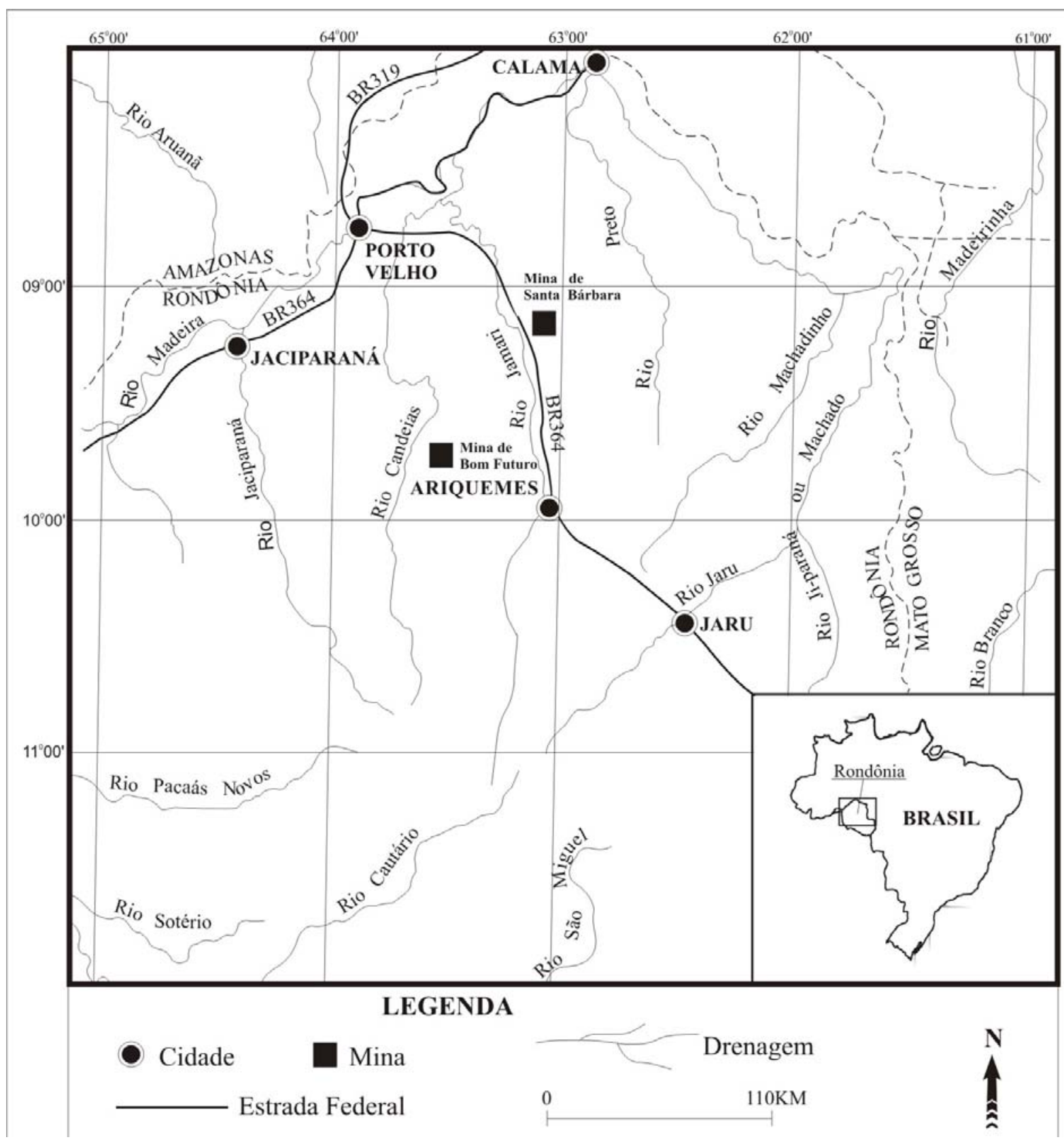

Figura 1 - Mapa de localização dos Distritos Mineiros de Santa Bárbara e Bom Futuro, Estado de Rondônia (adaptado de Leite Jr., 2002).

Figure 1 - Location map of Santa Bárbara and Bom Futuro Mining Districts, State of Rondônia (adapted from Leite Jr., 2002).

No depósito do tipo aluvionar, as rochas depositam-se nas planícies e vales de inundação rasos ou profundos, também denominado de paleovales. A cassiterita, proveniente das áreas fonte primárias, acumula-se devido as suas propriedades mineralógicas e aos processos de intemperismo, erosão e sedimentação. Esses depósitos também são conhecidos como depósitos de placers.

\section{MÉTODO GPR}

0 GPR - Ground Penetrating Radarou Georadar é um método eletromagnético que emprega ondas de rádio em altas freqüências (normalmente entre $10-1000 \mathrm{MHz}$ ), para localizar estruturas e feições geológicas rasas da subsuperfície ou localizar objetos enterrados pelo homem. 


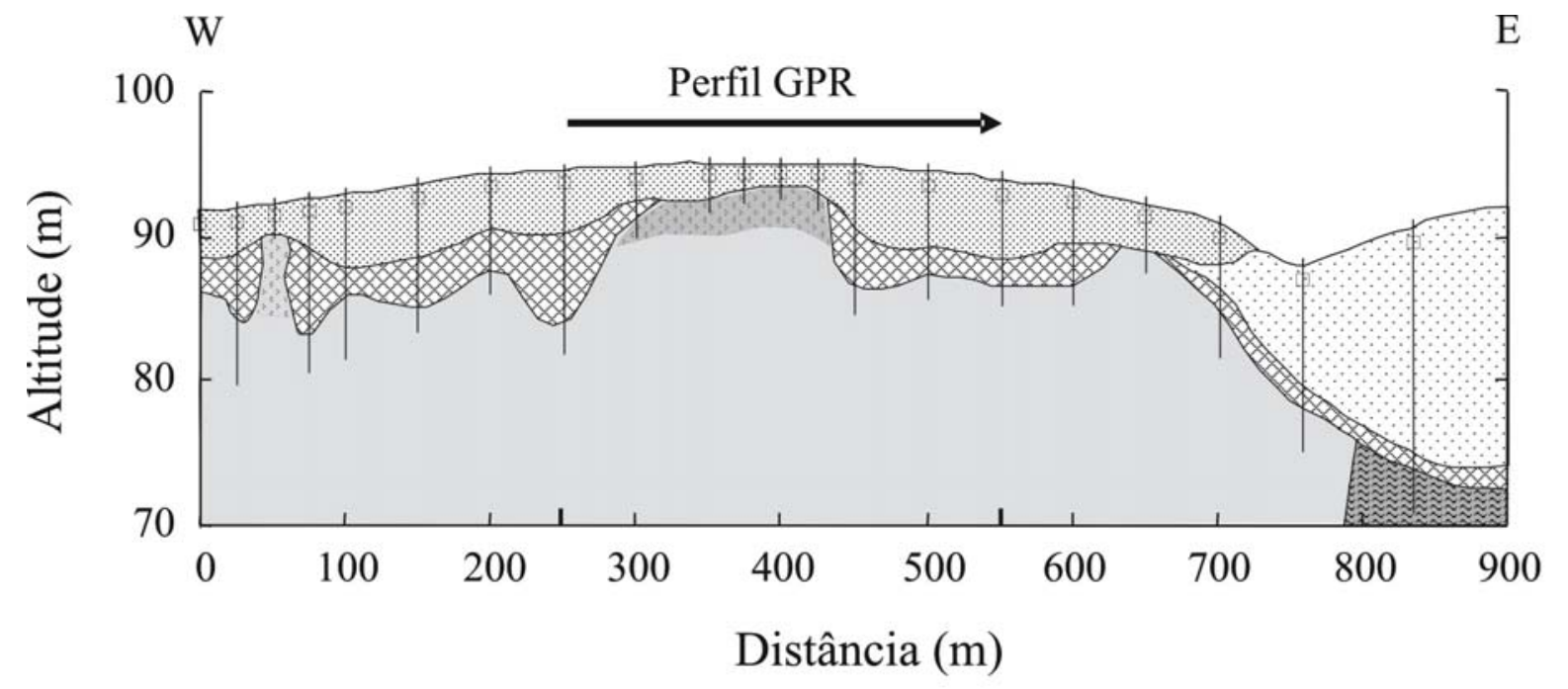

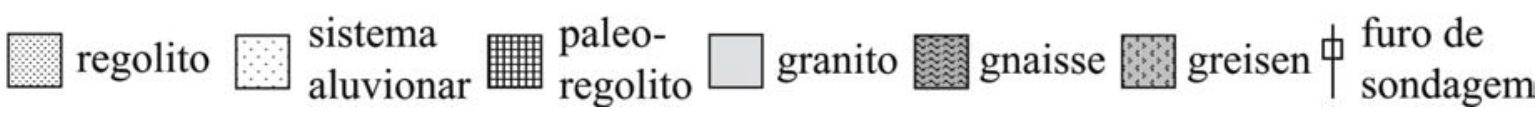

Figura 2 - Perfil geológico obtido de sondagens e localização do perfil GPR, Depósito Primário de Cortez, Mina de Santa Bárbara (adaptado de Mendonça, 2000). Figure 2 - Geologic profile obtained from boreholes and location of GPR profile, Cortez Primary Deposit, Santa Bárbara Mine (adapted from Mendonça, 2000).

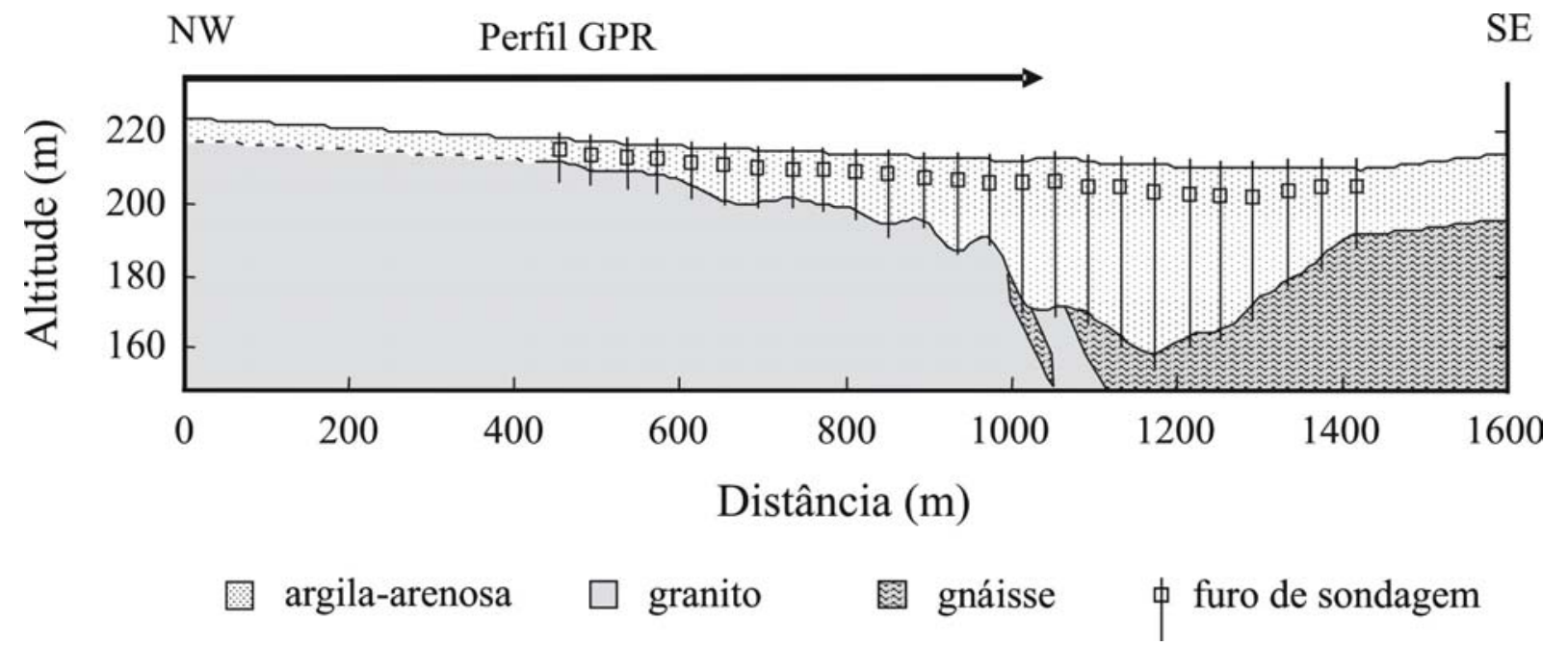

Figura 3 - Perfil geológico obtido de sondagens e localização do perfil GPR, Depósito Primário (de 0 a 400 m) e Depósito Secundário-Aluvionar (de $400 \mathrm{~m}$ em diante), Mina de Bom Futuro (adaptado de Mendonça, 2000).

Figure 3 - Geologic profile obtained from boreholes and location of GPR profile, Primary Deposit (0 to $400 \mathrm{~m}$ ) and Aluvionar-Secondary Deposit ( > 400 m), Bom Futuro Mine (adapted from Mendonça, 2000).

0 Georadar consiste em obter imagens de alta resolução da subsuperfície, através da transmissão de ondas eletromagnéticas (EM) de altas freqüências, que por sua vez é repetidamente radiada para dentro da terra por uma antena transmissora colocada na superfície (Davis \& Annan, 1989). A propagação da onda EM depende da freqüência do sinal transmitido e das propriedades elétricas dos materiais (condutividade elétrica, permissividade dielétrica), que são principalmente dependentes do conteúdo de água no solo (Topp et al., 1980).

As mudanças nas propriedades elétricas em subsuperfície fa- 


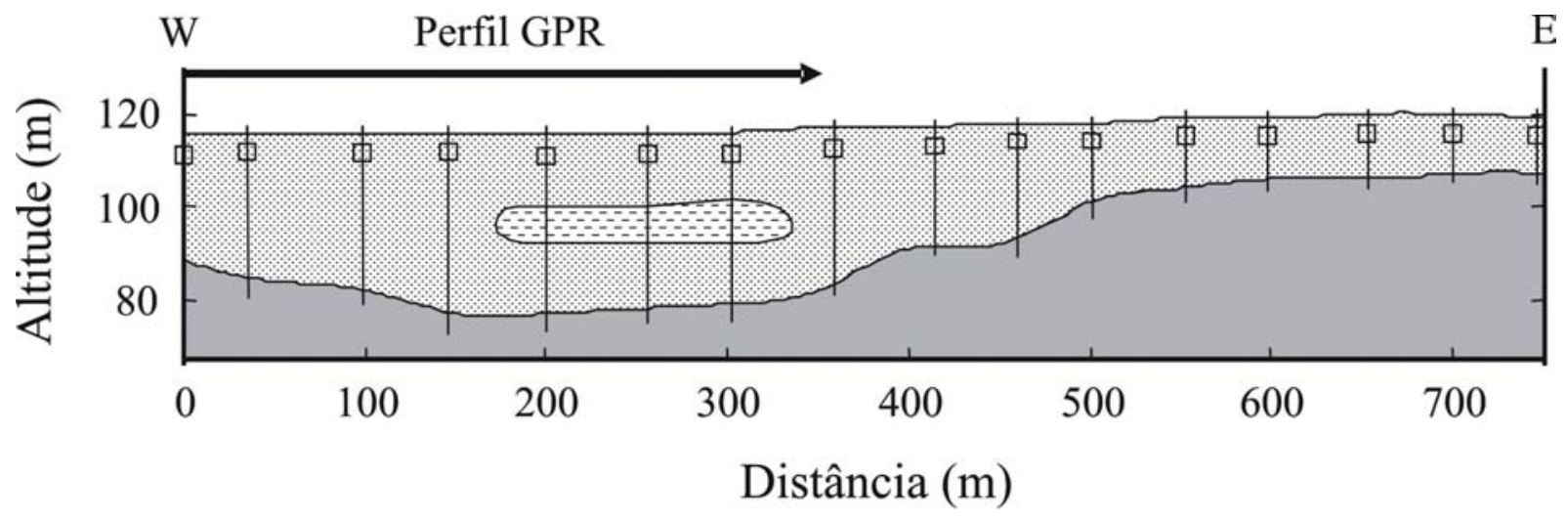

\section{argila-arenosa $\square$ granito $\quad$ argila plástica 中 furo de sondagem}

Figura 4 - Perfil geológico obtido de sondagens e localização do perfil GPR, Depósito Aluvionar de Taboquinha, Mina de Santa Bárbara (adaptado de Mendonça, 2000).

Figure 4 - Geologic profile obtained from boreholes and location of GPR profile, Aluvionar Taboquinha Deposit, Santa Bárbara Mine (adapted from Mendonça, 2000).

zem com que parte do sinal seja refletido. As ondas de radar refletidas e difratadas em diferentes interfaces são recebidas através de outra antena, denominada de antena receptora, também colocada na superfície do terreno.

A energia refletida é registrada em função do tempo de percurso (tempo de ida e volta), sendo amplificada, digitalizada e gravada no disco rígido de um laptop, deixando os dados prontos para o processamento posterior que se fizer necessário.

Informações detalhadas a respeito da propagação de ondas eletromagnéticas em altas freqüências, aquisição, processamento e interpretação de dados GPR podem ser encontradas na literatura apropriada (Ulriksen, 1982, Daniels, 1996; Porsani, 1999).

Quanto às aplicabilidades do método GPR, encontra-se na literatura as mais variadas aplicações em geologia (Porsani et al., 2004a), meio ambiente (Porsani et al., 2004b), geotecnia, planejamento urbano, arqueologia (Schimmel et al., 2003), dentre outras. 0 seu emprego visa principalmente localizar estruturas rasas da subsuperfície. Na exploração mineral o método GPR foi empregado visando:

i) Iocalização de depósitos de placers (Davis et al., 1984; Franco et al., 1997);

ii) localização de depósitos de sulfetos (Hammond \& Sprenke, 1991; Sigurdsson, 1994);

iii) no desenvolvimento de minas (Annan et al., 1988; Patterson \& Cook, 2002; Jha et al., 2004); iv) na localização de fraturas em rochas graníticas ornamentais (Botelho et al., 1999, Souza Jr. \& Porsani, 2001; Orlando, 2003) além de outras.

Uma nova potencialidade de sua aplicação é apresentada neste artigo, e está relacionada com a localização de depósitos primários e secundários de Cassiterita na região Amazônica.

\section{AQUISIÇÃO E PROCESSAMENTO DOS DADOS}

Os perfis GPR foram realizados nos distritos mineiros de Santa Bárbara e de Bom Futuro, áreas pertencentes à Companhia Estanífera do Brasil - CESBRA (Brascan Recursos Minerais S.A.) e à Empresa Brasileira de Estanho S.A. - EBESA (Paranapanema S.A.), respectivamente (Figura 1). Os trabalhos de campo concentraram-se nos depósitos estaníferos primários das minas de Santa Bárbara (Cortez I) e de Bom Futuro, e nos depósitos secundários aluvionares de Santa Bárbara (Taboquinha), ambas as minas são representativas da Província Estanífera de Rondônia.

0 trabalho de aquisição dos dados foi realizado no período de 10 a 18 de julho de 1999, totalizando 8 dias de campo. Foram adquiridos perfis de reflexão com afastamento constante e sondagens de velocidade do tipo WARR (Wide Angle Reflection and Refraction). Nos perfis de reflexão, a geometria das antenas transmissora e receptora é mantida com uma distância fixa constante, sendo transportada passo a passo ao longo do perfil. As antenas foram posicionadas transversais às orientações dos perfis, visando o máximo acoplamento entre o sinal trans- 
mitido e 0 sinal recebido. A Figura 5 mostra uma foto do arranjo das antenas de $25 \mathrm{MHz}$ durante a aquisição dos dados. 0 resultado obtido é uma imagem de alta resolução da subsuperfície, onde no eixo horizontal estão as posições das antenas (distâncias) e no eixo vertical, a energia da onda refletida é registrada em função do tempo de ida (sinal transmitido) e volta (sinal refletido). Os perfis estão apresentados em profundidade e a conversão tempo/profundidade está baseada na velocidade de 0,70 metros por nano segundos, i.e., $10^{-9}$ seg, obtida a partir das informações das sondagens. As sondagens de velocidade do tipo WARR não serão apresentadas devido à baixa qualidade dos resultados obtidos.

Os dados foram adquiridos com o equipamento sueco Ramac (Mala-GeoScience) pertencente ao Departamento de Geofísica do IAG/USP. Foram adquiridos sete perfis de reflexão GPR na mina de Santa Bárbara e seis perfis na mina de Bom Futuro, totalizando cerca de 4.870 metros lineares de investigação GPR. Os perfis foram obtidos utilizando-se as antenas de freqüências centrais de 25, 50 e $100 \mathrm{MHz}$. No perfil adquirido com as antenas de $100 \mathrm{MHz}$, a separação entre as antenas transmissora e receptora foi de 1 metro e o espaçamento entre cada traço de 0,5 metro; no perfil de $50 \mathrm{MHz}$, a separação entre as antenas foi de 2 metros e 0 espaçamento entre cada traço de 0,5 metro, e no perfil de $25 \mathrm{MHz}$, a separação entre as antenas foi de 4 metros e 0 espaçamento entre cada traço de 1 metro.

0 processamento dos dados GPR foi realizado com o programa Gradix (Interpex Limited, 1996). As principais etapas utilizadas foram: filtragem dewow (remoção dos ruídos de baixa freqüência), correção do tempo zero, filtragem temporal (filtro passa banda), ganhos no tempo (linear, esférico e exponencial), filtragem espacial (moving average de três traços), migração-fk e conversão tempo/profundidade (Yilmaz, 1987). Esses procedimentos são interativos e dependem de parâmetros fornecidos pelo intérprete. Neste trabalho, apresentaremos apenas os perfis que exibem resultados mais significativos.

\section{DISCUSSÃO E INTERPRETAÇÃO DOS RESULTADOS}

Os resultados obtidos foram interpretados com base nas seções geológicas obtidas através de sondagens. De um modo geral, os refletores observados nos perfis GPR foram correlacionados com os horizontes geológicos provenientes das sondagens. Dessa forma, as zonas de reflexões anômalas identificadas nos perfis GPR puderam ter um significado geológico quando comparadas com os perfis de sondagens.

Os perfis GPR foram realizados em depósitos primários e se- cundários. Nos depósitos primários, é importante determinar as zonas mais promissoras a mineralização, denominadas de zonas de greisens que aparecem cobertas por solo transportado ou residual com espessura mínima de dois $\mathrm{m}$. Para localizar estas zonas, foram realizados vários perfis GPR nos Distritos mineiros de Santa Bárbara e de Bom Futuro, utilizando-se as antenas de 25, 50 e $100 \mathrm{MHz}$

As Figuras 6 e 7 mostram os perfis GPR com $300 \mathrm{~m}$ de comprimento, obtidos no depósito primário de Cortez, Distrito de Santa Bárbara, com antenas de 100 e $50 \mathrm{MHz}$, respectivamente. Os perfis foram realizados sobre a mesma linha, visando obter boa resolução e boa penetração.

Nesses perfis, observa-se nitidamente uma zona com padrão de reflexão anômalo, entre as posições de 350 e 410 metros de distância, estendendo-se até cerca de 20 metros em profundidade. É provável que esta brusca mudança lateral no padrão de reflexão seja controlada por estruturas tectônicas como falhas e/ou fraturas preenchidas com água, pois o contraste entre as constantes dielétricas da água e da rocha encaixante, faz com que ocorra uma forte reflexão do sinal do GPR. Em depósitos estaníferos da região amazônica é comum a presença de óxidos de ferro preenchendo as fraturas, e este fato causa reflexões anômalas nos perfis GPR (Van Dam et al., 2002). No perfil geológico correspondente (Figura 2), nesta porção do perfil GPR ocorre uma zona de greisen. Na região anômala com 60 metros de extensão, o sinal do GPR alcança maiores profundidades de penetração em comparação com as rochas encaixantes, predominantemente argilosas. Este fato é devido à massa rochosa granítica que compõe 0 greisen ser bastante resistiva, proporcionando uma melhor penetração do sinal eletromagnético, ou seja, em meios resistivos ocorre pouca atenuação da onda eletromagnética do GPR.

As informações provenientes dos perfis GPR (Figuras 6 e 7) apresentam uma excelente concordância com as informaçõos obtidas do perfil geológico (Figura 2). Após a correlação entre a zona de reflexão anômala identificada nos perfis GPR com as informações provenientes do perfil geológico, pode-se afirmar que esta zona esteja relacionada com a zona mineralizada, denominada de zona de greisen.

No perfil de $50 \mathrm{MHz}$ (Figura 7), observa-se também refletores hiperbólicos com vértices nas posições de 0, 170, 220 e $280 \mathrm{~m}$ de distância e em torno de 8, 11, 6 e 9 metros de profundidade, respectivamente. Estes refletores são devidos às interferências das árvores de grande porte, comuns na região amazônica. Este fato foi comprovado pelo ajuste da velocidade da onda eletromagnética no ar $(v=0,3 m / n s)$ com os refletores hiperbólicos. 


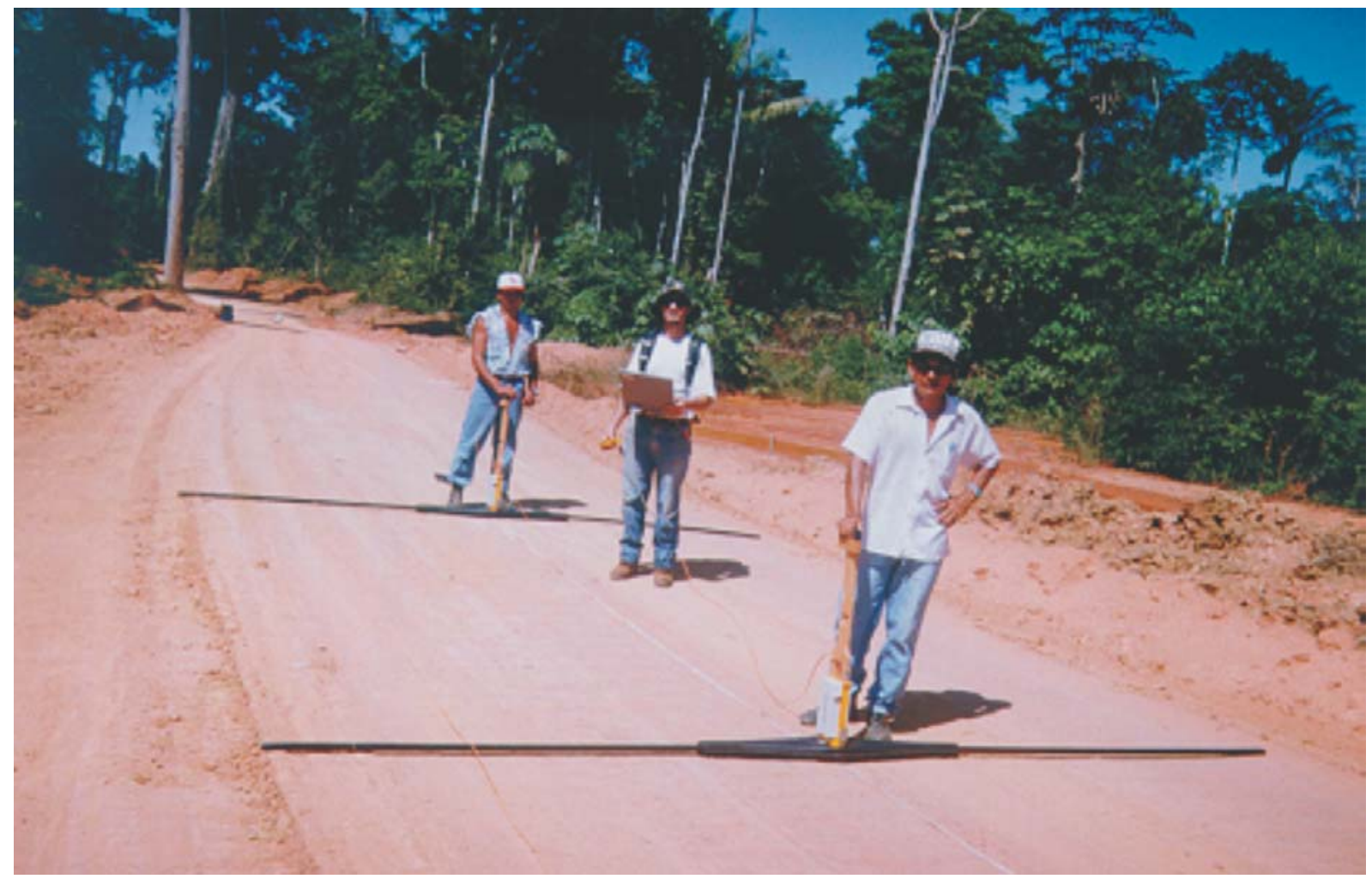

Figura 5 - Aquisição dos dados GPR com as antenas de $25 \mathrm{MHz}$.

Figure $\mathbf{5}$ - Acquisition of GPR data with $25 \mathrm{MHz}$ antennas.

\section{Distância (m)}

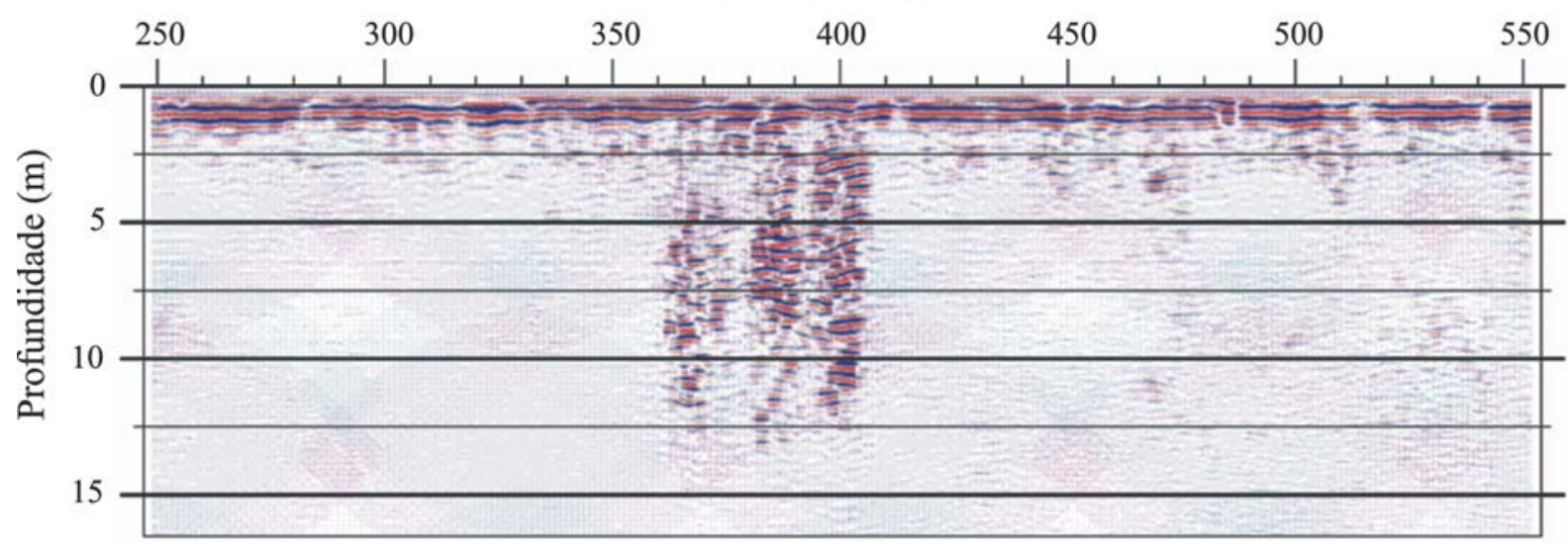

Figura 6 - Perfil GPR obtido com antenas de 100 MHz, Depósito Primário de Cortez, Mina de Santa Bárbara.

Figure 6 - GPR profile obtained with $100 \mathrm{MHz}$ antennas, Cortez Primary Deposit, Santa Bárbara Mine.

As Figuras 8,9 e 10 mostram os perfis GPR obtidos no Depósito primário de Bom Futuro, com antenas de 100, 50 e $25 \mathrm{MHz}$, respectivamente. 0 início desses perfis está próximo a Serra da Palanqueta e eles também foram realizados sobre a mesma linha, mudando-se apenas as freqüências das antenas, pois os resultados se complementam. 


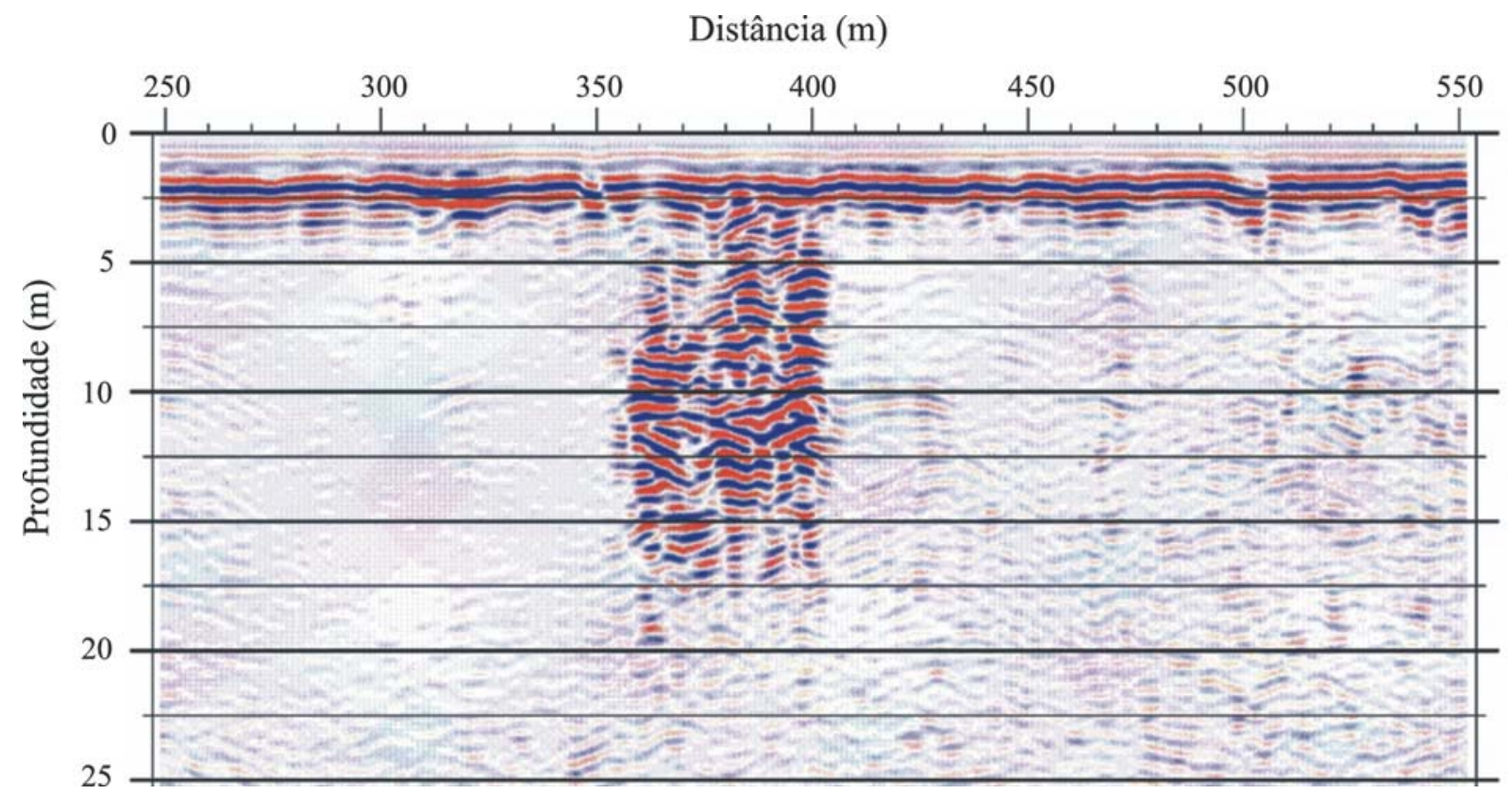

Figura 7 - Perfil GPR obtido com antenas de 50 MHz, Depósito Primário de Cortez, Mina de Santa Bárbara.

Figure 7 - GPR profile obtained with 50 MHz antennas, Cortez Primary Deposit, Santa Bárbara Mine.

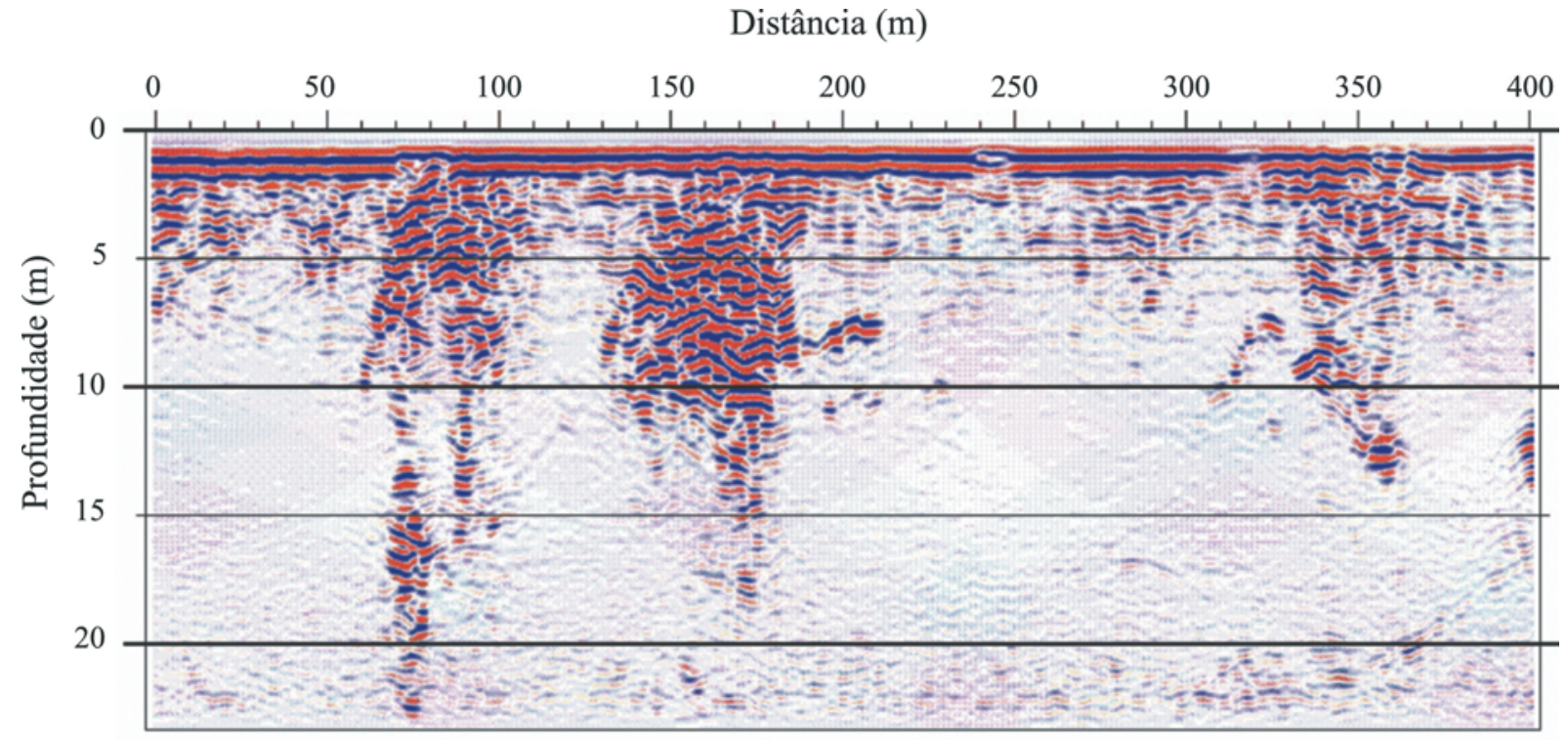

Figura 8 - Perfil GPR obtido com antenas de 100 MHz, Depósito Primário, Mina de Bom Futuro.

Figure 8 - GPR profile obtained with 100 MHz antennas, Primary Deposit, Bom Futuro Mine.

As Figuras 8 e 9 mostram os resultados dos perfis com 400 metros de comprimento e a Figura 10 mostra o resultado de um perfil com $1050 \mathrm{~m}$ de comprimento. Os primeiros 400 metros de comprimento destes perfis são caracterizados pelo depósito do tipo primário e a partir de 400 metros começa o depósito se- cundário do tipo aluvionar. Os resultados dos perfis GPR indicaram nitidamente três zonas de reflexão anômalas, caracterizadas por um padrão de reflexão caótico, entre as posições de 60-110, 130-190 e 310-370 metros de distância e até cerca de 30 metros de profundidade. Além dessas zonas, observa-se também um 


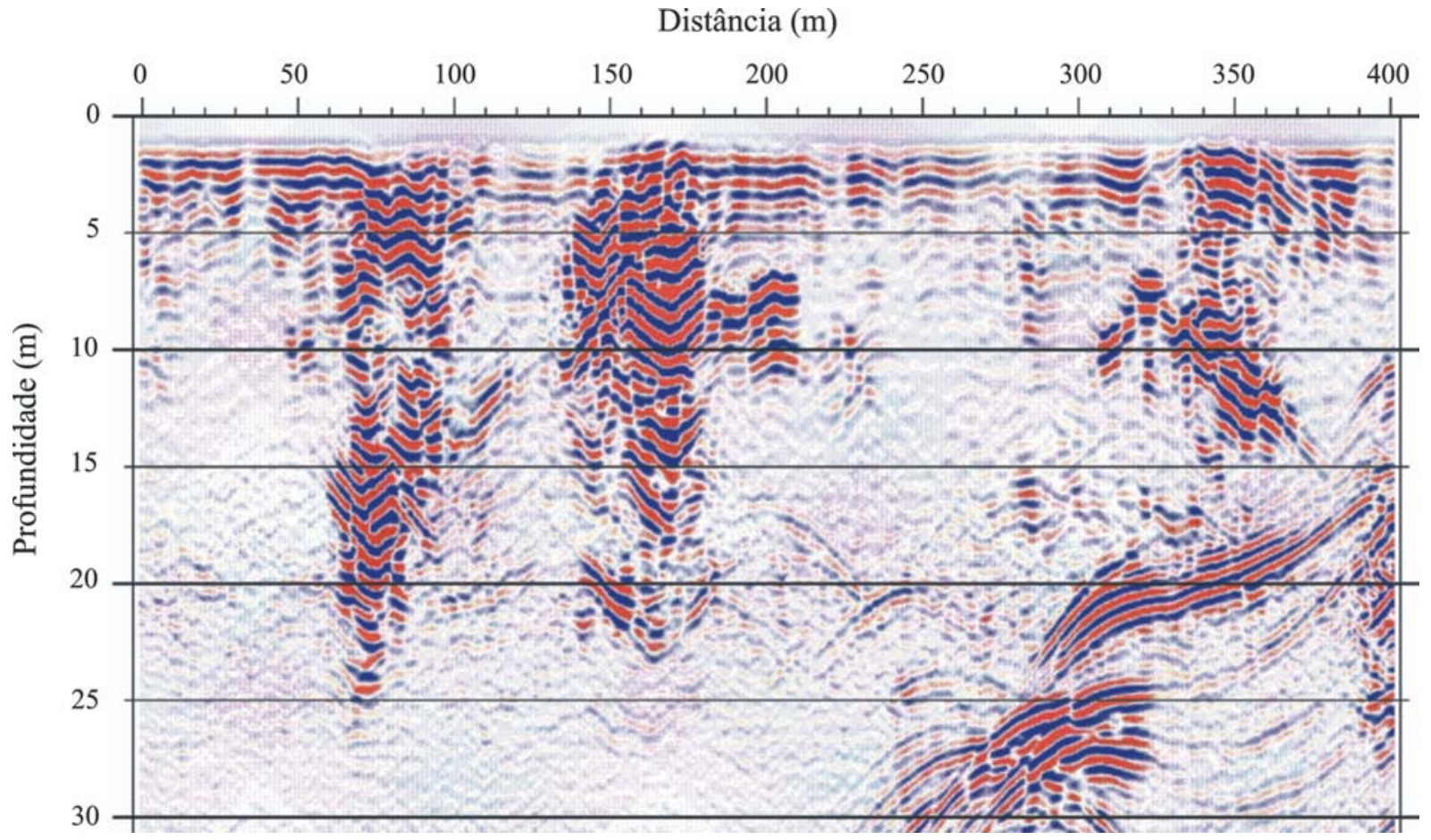

Figura 9 - Perfil GPR obtido com antenas de $50 \mathrm{MHz}$, Depósito Primário, Mina de Bom Futuro.

Figure 9 - GPR profile obtained with $50 \mathrm{MHz}$ antennas, Primary Deposit, Bom Futuro Mine.

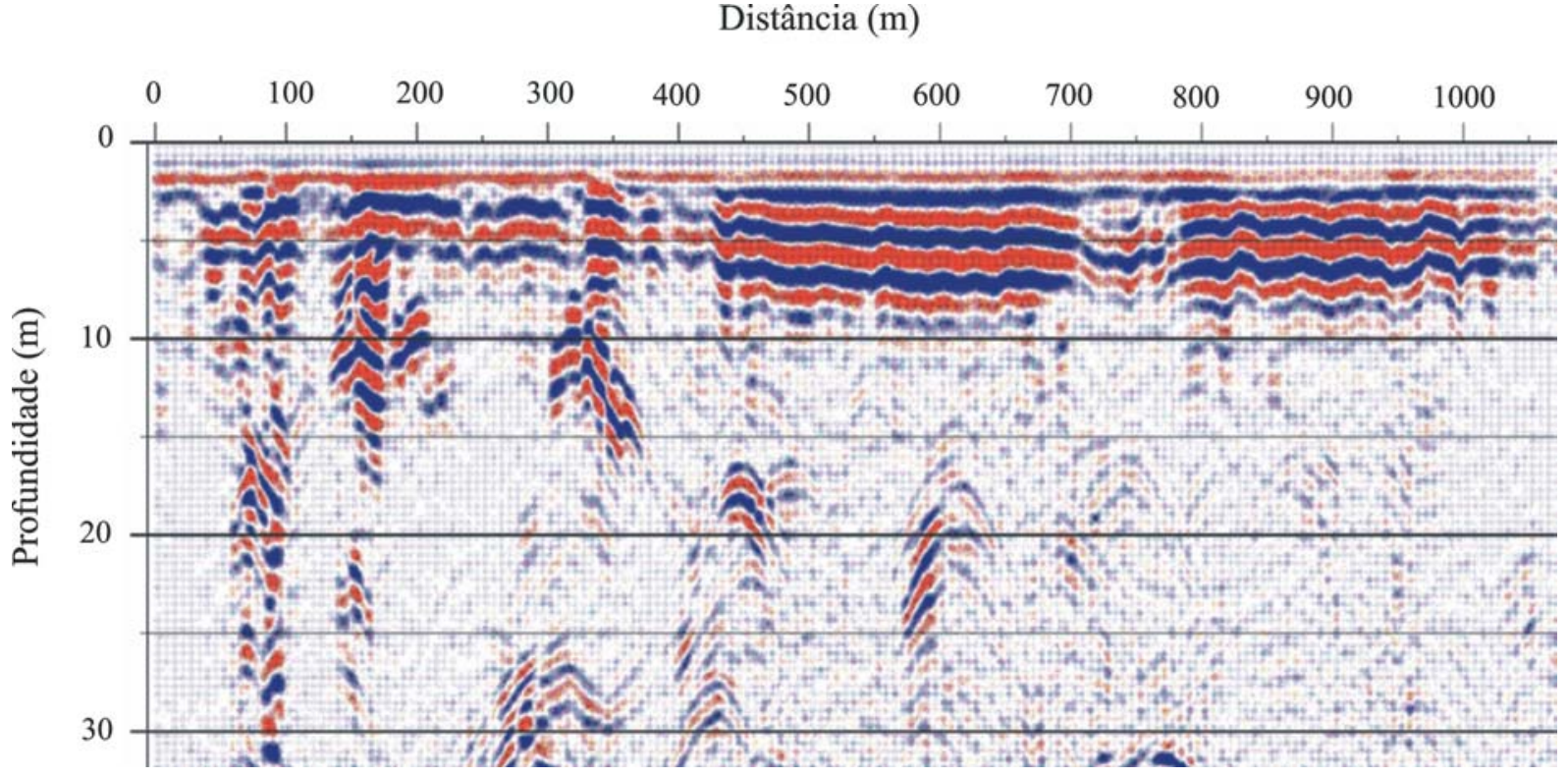

Figura 10 - Perfil GPR obtido com antenas de 25 MHz, Depósito Primário (de 0 a 400 m) e Depósito Secundário (de 400 m em diante), Mina de Bom Futuro.

Figure 10 - GPR profile obtained with $25 \mathrm{MHz}$ antennas, Primary Deposit (0 to $400 \mathrm{~m}$ ) and Secondary Deposit ( > $400 \mathrm{~m}$ ), Bom Futuro Mine.

forte refletor subhorizontal entre as posições de 190 e 210 metros de distância e em torno de 7 metros de profundidade, estando provavelmente relacionado com fraturas subhorizontais.

Nas Figuras 9 e 10 observam-se também vários refletores hi- 


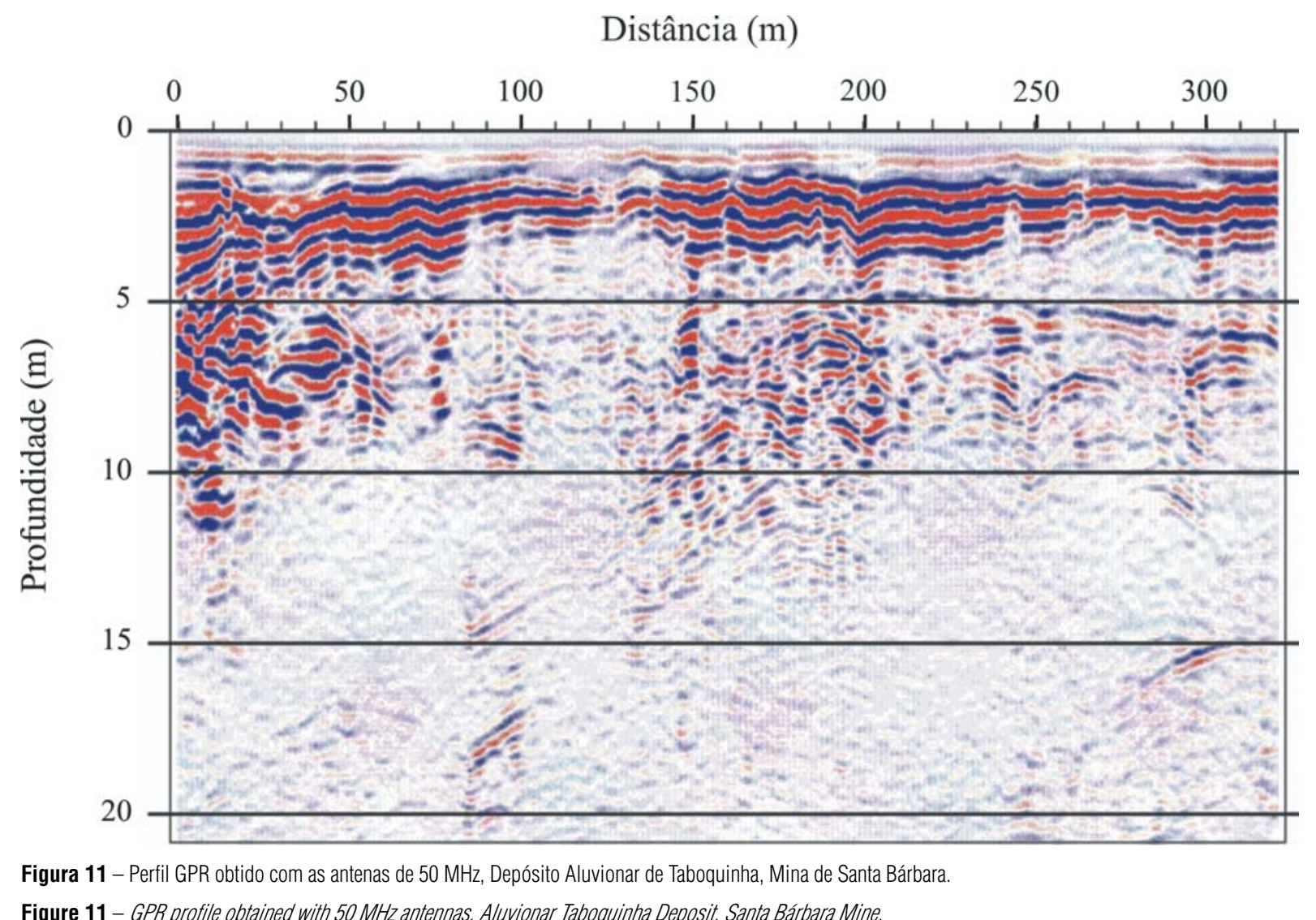

perbólicos entre 200-800 metros de distância e a partir de 15 metros de profundidade. Este fato deve-se à reflexão da onda eletromagnética aérea nas árvores de grande porte. Estes refletores são indesejáveis e não devem ser considerados na interpretação dos perfis GPR. Por outro lado, na Figura 10 entre as posições de 700 e 780 metros de distância ocorre um refletor curvo raso de bastante interesse para a prospecção de cassiterita, estando relacionado com uma estrutura de paleocanal. Esta é uma importante estrutura encontrada nos depósitos secundários, pois devido à cassiterita ser um resistato de elevada densidade, ela é transportada do depósito primário (fonte do minério) até ser depositada em paleocanais, também conhecido como depósitos de placers.

É importante ressaltar que nos primeiros 400 metros desses perfis não existem informações de sondagens, e portanto, as informações fornecidas pelos perfis GPR são de fundamental importância para a exploração mineral, mostrando que as investigações indiretas podem contribuir de maneira significativa no processo exploratório de cassiterita na região amazônica.

0 uso integrado dos perfis GPR realizados nos depósitos primários com os perfis geológicos obtidos a partir da correlação litológica entre as sondagens, permitiu delimitar zonas mineralizadas do tipo greisens em subsuperfície. É provável que as zonas de greisens estejam associadas às estruturas tectônicas como falhas/fraturas e zonas de cisalhamentos, devido à brusca variação lateral nas propriedades elétricas imageadas pelo GPR. Os padrões de reflexão anômalos encontrados nos perfis realizados na Mina de Bom Futuro apresentaram as mesmas características dos perfis realizados na Mina de Santa Bárbara, sugerindo ser esse o padrão de reflexão típico de zonas greisenizadas rasas. Esses resultados apresentam uma excelente concordância com as informações geológicas obtidas diretamente das sondagens.

A Figura 11 mostra um perfil GPR obtido com as antenas de $50 \mathrm{MHz}$ no Depósito Aluvionar de Taboquinha, na Mina de Santa Bárbara. Devido à camada superficial ser bastante argilosa, a profundidade de penetração das ondas de radar fica limitada aos primeiros 10 metros de profundidade. Apesar disso, pode-se identificar um refletor ligeiramente inclinado entre as posições de $200 \mathrm{e}$ 320 metros de distância, e em torno de 6 metros de profundidade, que está relacionado com a profundidade do topo do nível freático. 
Esta informação foi comprovada com base nas informações obtidas diretamente das sondagens. Observam-se também alguns refletores hiperbólicos que estão relacionados com as interferências superficiais provenientes de árvores de grande porte.

\section{CONCLUSÕES}

Os resultados dos perfis GPR realizados nas minas de Santa Bárbara e de Bom Futuro apresentaram uma excelente concordância com as informações geológicas provenientes das sondagens.

Em relação aos depósitos primários das minas de Santa Bárbara e de Bom Futuro, o método GPR permitiu identificar zonas com predominância de corpos de greisens até $30 \mathrm{~m}$ de profundidade, caracterizadas por um elevado padrão de reflexão caótico e irregular. As características similares dessas reflexões sugerem ser esse o padrão de reflexão típico de zonas greisenizadas na Província Estanífera de Rondônia.

No depósito secundário aluvionar da mina de Bom Futuro, 0 método GPR permitiu localizar um paleocanal, que é uma importante estrutura para a prospecção de cassiterita em depósitos de placers.

Demonstra-se, assim, a aplicabilidade do método GPR para a localização de mineralizações estaníferas primárias e paleocanais, e portanto, o seu emprego poderá ser extrapolado para ambientes similares do Cráton Amazônico.

Os resultados obtidos sugerem que o método GPR poderá ser utilizado como etapa de reconhecimento, antecedendo a realização das sondagens. Este fato permitirá otimizar os custos envolvidos na etapa da prospecção, diminuindo o número de sondagens no processo exploratório.

\section{AGRADECIMENTOS}

Ao Departamento de Geofísica do IAG/USP pela infra-estrutura necessária para a realização desta pesquisa. Ao PADCT/CNPq (Processo-62.0152/98.2) pelo apoio financeiro para a execução deste projeto de pesquisa. Às mineradoras CESBRA e EBESA pelos apoios logísticos. Aos alunos da graduação de Geologia (UFMT) e aos ajudantes cedidos pelas mineradoras, que foram fundamentais para a aquisição dos dados.

\section{REFERÊNCIAS}

ANNAN AP, DAVIS JL \& GENDZWILL D. 1988. Radar sounding in potash mines, Saskatchewan, Canada. Geophysics, 53(12): 1556-1564.

BETTENCOURT JS, LEITE JR. WB, PAYOLLA BL, SCANDOLARA JE, MUZZOLON R \& VIAN JAJ. 1997. The rapakivi granites of the Rondônia
Tin Province, Northern Brazil. In: International Symposium on Granites and Associated Mineralizations, Salvador, Bahia, Brazil, August 24-29, p. 1-31.

BOTELHO MAB, CERQUEIRA NETO JXC \& ARANHA P. 1999. Estudo sobre a economicidade de jazidas de rochas ornamentais empregando 0 Geo-Radar (GPR). In: $6^{\circ}$ International Congress of the Brazilian Geophysical Society, Rio de Janeiro, resumos expandidos, cd-room.

DALL'IGNA LF. 1996. A mineração e 0 garimpo de cassiterita em Rondônia. Revista da CPRM, 1: 56-61.

DANIELS JJ. 1996. Surface Penetrating Radar. The Institution of Eletrical Engineers, London, United Kingdom, $300 \mathrm{p}$.

DAVIS JL, ANNAN AP \& VAUGHAN CJ. 1984. Placer exploration using radar and seismic methods. In: International Congress of Society of Exploration Geophysical, 54, 1984, Atlanta. Expanded Abstracts, Atlanta: SEG, p. 67-72.

DAVIS JL \& ANNAN AP. 1989. Ground penetrating radar for high resolution mapping of soil and rock stratigraphy. Geophysical Prospecting, 37: 531-551.

FRANCO HA, MELLO GA, PIRES ACB \& COSTA ALL. 1997. Aplicação das metodologias GPR e EM34-3XL em depósitos aluvionares do vale do Rio do Sono, Novo Acordo-TO. In: $5^{\circ}$ International Congress of the Brazilian Geophysical Society, Resumos Expandidos, São Paulo: SBGt, 1: $526-529$.

HAMMOND WR \& SPRENKE KF. 1991. Radar detection of subglacial sulfides. Geophysics, 56(6): (june), 870-873.

INTERPEX LIMITED. 1996. GRADIX V1 Ground Penetration Radar Processing and Interpretation. Manual de Processamento e Interpretação, Golden, Colorado, USA, 222 p.

JHA PC, BALASUBRAMANIAM VR, SANDEEP N, SIVARAM YV \& GUPTA RN. 2004. GPR applications in mapping barrier thickness in coal mines: Some case studies. In: Proceedings of the Tenth International Conference on Ground Penetrating Radar (GPR'2004), Delft, The Netherlands, cd-room.

LEITE JR. WB. 2002. A suíte intrusiva Santa Clara (R0) e a mineralização primária polimetálica (Sn, W, Nb, Ta, Zn, Cu, Pb) associada. Tese de Doutorado, Instituto de Geociências da Universidade de São Paulo, 303 p.

MENDONÇA CA. 2000. Investigações geofísicas na província estanífera de Rondônia: distritos de Bom Futuro e Santa Bárbara. Relatório parcial do projeto PADCT-III/GTM (Processo-62.0152/98.2).

ORLANDO L. 2003. Semiquantitative evaluation of massive rock quality using ground penetrating radar. Journal of Applied Geophysics, 52: 1-9.

PATTERSON JE \& COOK FA. 2002. Successful application of groundpenetrating radar in the exploration of gem tourmaline pegmatites of southern California. Geophysical Prospecting, 50(2): 107-117. 
PORSANI JL. 1999. Ground Penetrating Radar (GPR): Proposta metodológica de emprego em estudos geológico-geotécnicos nas regiões de Rio Claro e Descalvado - SP. Tese de Doutorado, Instituto de Geociências e Ciências Exatas, UNESP - Campus de Rio Claro - SP, 145 p.

PORSANI JL, BORGES WR, ELIS VR, DIOGO LA, HIODO FY, MARRANO A \& BIRELLI CA. 2004a. Investigações Geofísicas de Superfície e de Poço no Sítio Controlado de Geofísica rasa do IAG/USP. Revista Brasileira de Geofísica (no Prelo)

PORSANI JL, MALAGUTTI FILHO W, ELIS VR, FISSEHA S, DOURADO JC \& MOURA HP. 2004b. The use of GPR and VES in delineating a contamination plume in a Landfill site: a case study in SE Brazil. Journal of Applied Geophysics, 55(3-4): 199-209.

SCHIMMEL PB, PORSANI JL, FIGUTI L \& De BLASIS P. 2003. Aplicação de Métodos Geofísicos em Arqueologia: Primeiros Resultados Obtidos no Sambaqui Fluvial Capelinha, Cajati-SP, Brasil. Revista Brasileira do Museu de Arqueologia e Etnologia da USP, São Paulo, n.2, p. 43-54.

SIGURDSSON T. 1994. Application of GPR for geological mapping, exploration of industrial mineralization and sulphide deposits. In: Interna- tional Conference on Ground Penetrating Radar, 5, 1994, Ontario. Proceedings, Ontario: GPR'94, p. 941-955.

SOUZA JR. AO \& PORSANI JL. 2001. Localização de fraturas em rochas graníticas, no município de Capão Bonito - SP, Brasil. In: $7^{0}$ International Congress of the Brazilian Geophysical Society, Resumos Expandidos, Salvador, BA, SBGf, cd-room.

TOPP GC, DAVIS JL \& ANNAN AP. 1980. Electromagnetic determination of soil water content: measurements in coaxial transmission lines. Water Resources Research, 16(3): 574-582.

ULRIKSEN CPF. 1982. Application of impulse radar to civil engineering. Doctoral Thesis - Lund University of Technology, 179 p.

VAN DAM RL, SCHLAGER W, DEKKERS MJ \& HUISMAN JA. 2002. Iron oxides as a cause of GPR reflections. Geophysics, 67(2): (march-april), 536-545.

YILMAZ 0. 1987. Seismic data processing. Tulsa: Society of Exploration Geophysics Press, 526 pp.

\section{NOTAS SOBRE OS AUTORES}

Jorge Luís Porsani. Geólogo pelo Instituto de Geociências da UFBa (1987). Mestrado em Geofísica pelo Núcleo de Pesquisas Geofísicas Aplicadas à Prospecção de Hidrocarbonetos da UFPa (1991). De 1991 a 1996, trabalhou como Geofísico no Centro de Pesquisas da PETROBRAS. Doutorado em Geociências e Meio Ambiente pelo Instituto de Geociências e Ciências Exatas da UNESP (1999). Desde 01/12/1998 é Docente do Departamento de Geofísica do IAG/USP, atuando com métodos geoelétricos e eletromagnéticos aplicados à geologia, geotecnia, exploração mineral, meio ambiente e arqueologia. Atualmente, é Vice Coordenador do Programa de Pós-Graduação em Geofísica e Coordenador do Projeto de Instalação do Sítio Controlado de Geofísica Rasa do IAG (Fapesp 02/07509-1).

Carlos Alberto Mendonça. Formado em geologia pela Universidade de São Paulo em 1985 e doutorado em geofísica pela Universidade Federal do Pará, em 1992. Trabalhou em empresas de prospecção mineral de 1985 a 1988 e desde 1993 é professor de geofísica aplicada no Departamento de Geofísica do IAG/USP. Seus campos de interesse envolvem a aplicação integrada de métodos geofísicos (geoelétricos, magnéticos e gravimétricos) na investigação de problemas relacionados à prospecção de recursos naturais e estudos arqueológicos.

Jorge Silva Bettencourt. Geólogo, Doutor (1972), Livre Docente (1992), pós-doutor pela Université Pierre e Marie Currie, Paris, França (1975-1976) e Professor Titular (1993) pelo IGc-USP. É, atualmente, Professor Titular (permissionário) do Departamento de Mineralogia e Geotectônica do IGc-USP. Trabalhou, alternadamente, em várias companhias mineiras e na universidade, desde 0 início da carreira profissional. Realiza pesquisas em metalogenia, modelagem de depósitos minerais e exploração mineral sendo, também, consultor de várias companhias mineiras. É assessor científico da FAPESP, CNPq, CAPES e FINEP.

Francisco Yukio Hiodo. Graduado em Física pelo Instituto de Física da Universidade de São Paulo-USP em 1972. Mestrado em Geofísica pelo Instituto de Astronomia, Geofísica e Ciências Atmosféricas (IAG-USP) em 1981. Doutorado em Geofísica pelo IAG-USP em 1990. Auxiliar de Ensino no IAG-USP em 1973. Professor Assistente em 1981. Professor Doutor do IAG-USP desde 1990. Atualmente é especialista em Instrumentação Geofísica nas áreas de: Paleomagnetísmo (magnetômetros rotativos e desmagnetizadores), Geofísica Nuclear (espectrômetros gama e emanômetros de radônio), Perfilagem de poços tubulares, Geotermometria e sensores de temperatura, Magnetômetros de precessão de prótons e fluxgate, Métodos geoelétricos e eletromagnéticos.

José Antônio Jeronymo Vian. Geólogo pela UNESP de Rio Claro (1976). Atuou como Geólogo Chefe de Campo em várias empresas: Grupo Brumadinho Mineração Oriente Novo (1977 a 1978); Sondasa Eng. (1978 a 1981); Engessonda (1981); CESBRA/BRASCAN (1984 a 1990), entre 1984 e 1986 atuou na exploração de Au e Sn, a partir de 1990 foi supervisor de Planejamento da Mina Santa Bárbara-RO; Sergeo Consultoria (1990 a 1993) elaborou e acompanhou EIA, RIMA e DNPMs; CESBRA/BRASCAN (1993 até 2004) atua como Geólogo Chefe na Exploração Regional para Au e Sn no AM, RO, G0 e PA, e Gerência de Geologia e Meio Ambiente do Grupamento Mineiro Santa Bárbara.

Jorge Eduardo da Silva. Bacharel em Geofísica pelo Instituto de Astronomia, Geofísica e Ciências Atmosféricas da Universidade de São Paulo (IAG - USP) em 2003. Atualmente trabalha como Geofísico na área de aquisição, processamento e interpretação de dados geofísicos junto à empresa AeroGeoPhysica Latinoamerica (AGP-LA). 